\title{
Existence conditions for symmetric generalized quasi-variational inclusion problems
}

Nguyen Van Hung*

\section{"Correspondence: ngvhungdhdt@yahoo.com Department of Mathematics, Dong Thap University, 783 Pham Huu Lau Street, Ward 6, Cao Lanh City, Vietnam}

\begin{abstract}
In this paper, we establish an existence theorem by using the Kakutani-Fan-Glicksberg fixed-point theorem for a symmetric generalized quasi-variational inclusion problem in real locally convex Hausdorff topological vector spaces. Moreover, the closedness of the solution set for this problem is obtained. As special cases, we also derive the existence results for symmetric weak and strong quasi-equilibrium problems. The results presented in the paper improve and extend the main results in the literature.

MSC: $90 \mathrm{~B} 20 ; 49 J 40$

Keywords: symmetric generalized quasi-variational inclusion problem; symmetric weak quasi-equilibrium problem; symmetric strong quasi-equilibrium problem; Kakutani-Fan-Glicksberg fixed-point theorem; existence; closedness
\end{abstract}

\section{Introduction}

Let $X$ and $Z$ be real locally convex Hausdorff spaces, $A \subset X$ be a nonempty subset and $C \subset Z$ be a closed convex pointed cone. Let $F: A \times A \rightarrow 2^{Z}$ be a given set-valued mapping. Ansari et al. [1] introduced the following two problems (in short, (VEP) and (SVEP)), respectively:

Find $\bar{x} \in A$ such that

$$
F(x, y) \not \subset-\operatorname{int} C, \quad \forall y \in A,
$$

and find $\bar{x} \in A$ such that

$$
F(x, y) \subset C, \quad \forall y \in A .
$$

The problem (VEP) is called the weak vector equilibrium problem and the problem (SVEP) is called the strong vector equilibrium problem. Later, these two problems have been studied by many authors; see, for example, $[2,3]$ and references.

In 2008, Long et al. [4] introduced a generalized strong vector quasi-equilibrium problem (for short, (GSVQEP)). Let $X, Y$ and $Z$ be real locally convex Hausdorff topological vector spaces, $A \subset X$ and $B \subset Y$ be nonempty compact convex subsets and $C \subset Z$ be a nonempty closed convex cone, and let $S: A \rightarrow 2^{A}, T: A \rightarrow 2^{B}, F: A \times B \times A \rightarrow 2^{Z}$ be set-valued mappings.

(c) 2013 Hung; licensee Springer. This is an Open Access article distributed under the terms of the Creative Commons Attribution License (http://creativecommons.org/licenses/by/2.0), which permits unrestricted use, distribution, and reproduction in any medium, provided the original work is properly cited. 
(GSVQEP): Find $\bar{x} \in A$ and $\bar{y} \in T(\bar{x})$ such that $\bar{x} \in S(\bar{x})$ and

$$
F(\bar{x}, \bar{y}, x) \subset C, \quad \forall x \in S(\bar{x}),
$$

where $\bar{x}$ is a strong solution of (GSVQEP).

Recently, Plubtieng and Sitthithakerngkietet [5] considered the system of generalized strong vector quasi-equilibrium problems (in short, (SGSVQEPs)). This model is a general problem which contains (GVEP) and (QSVQEP). Let $X, Y, Z$ be real locally convex Hausdorff topological vector spaces, $A \subset X$ and $B \subset Y$ be nonempty compact convex subsets and $C \subset Z$ be a nonempty closed convex cone. Let $S_{1}, S_{2}: A \rightarrow 2^{A}, T_{1}, T_{2}: A \rightarrow 2^{B}$ and $F_{1}, F_{2}: A \times B \times A \rightarrow 2^{Z}$ be set-valued mappings. They considered (SGSVQEPs) as follows.

Find $(\bar{x}, \bar{u}) \in A \times A$ and $\bar{z} \in T_{1}(\bar{x}), \bar{v} \in T_{2}(\bar{u})$ such that $\bar{x} \in S_{1}(\bar{x}), \bar{u} \in S_{2}(\bar{u})$ and

$$
F_{1}(\bar{x}, \bar{z}, y) \subset C, \quad \forall y \in S_{1}(\bar{x})
$$

and

$$
F_{2}(\bar{u}, \bar{v}, y) \subset C, \quad \forall y \in S_{2}(\bar{u}),
$$

where $(\bar{x}, \bar{u})$ is a strong solution of (SGSVQEPs).

Very recently, new symmetric strong vector quasi-equilibrium problems (in short, (SSVQEP)) in Hausdorff locally convex spaces were introduced by Chen et al. [6]. Let $X, Y, Z$ be real locally convex Hausdorff topological vector spaces, $A \subset X$ and $B \subset Y$ be nonempty compact convex subsets and $C \subset Z$ be a nonempty closed convex cone. Let $S_{1}, S_{2}: A \times A \rightarrow 2^{A}, T_{1}, T_{2}: A \times A \rightarrow 2^{B}$ and $F_{1}, F_{2}: A \times B \times A \rightarrow 2^{Z}$ be set-valued mappings. They considered the following (SSVQEP):

Find $(\bar{x}, \bar{u}) \in A \times A$ and $\bar{z} \in T_{1}(\bar{x}, \bar{u}), \bar{v} \in T_{2}(\bar{x}, \bar{u})$ such that $\bar{x} \in S_{1}(\bar{x}, \bar{u}), \bar{u} \in S_{2}(\bar{x}, \bar{u})$ and

$$
F_{1}(\bar{x}, \bar{z}, y) \subset C, \quad \forall y \in S_{1}(\bar{x}, \bar{u})
$$

and

$$
F_{2}(\bar{u}, \bar{v}, y) \subset C, \quad \forall y \in S_{2}(\bar{x}, \bar{u})
$$

where $(\bar{x}, \bar{u})$ is a strong solution for the (SSVQEP).

Motivated by the research works mentioned above, in this paper, we introduce symmetric generalized quasi-variational inclusion problems. Let $X, Y, Z$ be real locally convex Hausdorff topological vector spaces and $A \subset X, B \subset Y$ be nonempty compact convex subsets. Let $K_{i}, P_{i}: A \times A \rightarrow 2^{A}, T_{i}: A \times A \rightarrow 2^{B}$ and $F_{i}: A \times B \times A \rightarrow 2^{Z}, i=1,2$, be set-valued mappings.

Now, we adopt the following notations (see [7-9]). Letters w, $m$ and s are used for weak, middle and strong problems, respectively. For subsets $U$ and $V$ under consideration, we 
adopt the following notations:

$$
\begin{array}{ll}
(u, v) \mathrm{w} U \times V \quad \text { means } \forall u \in U, \exists v \in V, \\
(u, v) \mathrm{m} U \times V \quad \text { means } \exists v \in V, \forall u \in U, \\
(u, v) \mathrm{s} U \times V \quad \text { means } \forall u \in U, \forall v \in V .
\end{array}
$$

Let $\alpha \in\{\mathrm{w}, \mathrm{m}, \mathrm{s}\}$. We consider the following for symmetric generalized quasi-variational inclusion problem (in short, $\left(\mathrm{SQVIP}_{\alpha}\right)$ ).

$\left(\operatorname{SQVIP}_{\alpha}\right)$ : Find $(\bar{x}, \bar{u}) \in A \times A$ such that $\bar{x} \in K_{1}(\bar{x}, \bar{u}), \bar{u} \in K_{2}(\bar{x}, \bar{u})$ and

$$
\begin{array}{ll}
(y, \bar{z}) \alpha P_{1}(\bar{x}, \bar{u}) \times T_{1}(\bar{x}, \bar{u}) & \text { satisfying } 0 \in F_{1}(\bar{x}, \bar{z}, y), \\
(y, \bar{v}) \alpha P_{2}(\bar{x}, \bar{u}) \times T_{2}(\bar{x}, \bar{u}) & \text { satisfying } 0 \in F_{2}(\bar{u}, \bar{v}, y) .
\end{array}
$$

We denote that $\Xi_{\alpha}(F)$ is the solution set of $\left(\operatorname{SQVIP}_{\alpha}\right)$.

The symmetric generalized quasi-variational inclusion problems include as special cases symmetric generalized vector quasi-equilibrium problems, vector quasi-equilibrium problems, symmetric vector quasi-variational inequality problems, variational relation problems, etc. In recent years, a lot of results for the existence of solutions for symmetric vector quasi-equilibrium problems, vector quasi-equilibrium problems, vector quasivariational inequality problems, variational relation problems and optimization problems have been established by many authors in different ways. For example, equilibrium problems [1-6, 10-21], variational inequality problems [22-24], variational relation problems $[7,25]$, optimization problems $[22,26]$ and the references therein.

The structure of our paper is as follows. In the first part of this article, we introduce the model symmetric generalized quasi-variational inclusion problem. In Section 2, we recall definitions for later use. In Section 3, we establish an existence and closedness theorem by using the Kakutani-Fan-Glicksberg fixed-point theorem for a symmetric generalized quasi-variational inclusion problem. Applications to symmetric weak and strong vector quasi-equilibrium problems are presented in Section 4.

\section{Preliminaries}

In this section, we recall some basic definitions and some of their properties.

Definition $1[27,28]$ Let $X, Y$ be two topological vector spaces, $A$ be a nonempty subset of $X$ and $F: A \rightarrow 2^{Z}$ be a set-valued mapping.

(i) $F$ is said to be lower semicontinuous (lsc) at $x_{0} \in A$ if $F\left(x_{0}\right) \cap U \neq \emptyset$ for some open set $U \subseteq Y$ implies the existence of a neighborhood $N$ of $x_{0}$ such that $F(x) \cap U \neq \emptyset$, $\forall x \in N$. $F$ is said to be lower semicontinuous in $A$ if it is lower semicontinuous at all $x_{0} \in A$.

(ii) $F$ is said to be upper semicontinuous (usc) at $x_{0} \in A$ if for each open set $U \supseteq G\left(x_{0}\right)$, there is a neighborhood $N$ of $x_{0}$ such that $U \supseteq F(x), \forall x \in N$. $F$ is said to be upper semicontinuous in $A$ if it is upper semicontinuous at all $x_{0} \in A$.

(iii) $F$ is said to be continuous in $A$ if it is both lsc and usc in $A$.

(iv) $F$ is said to be closed if $\operatorname{Graph}(F)=\{(x, y): x \in A, y \in F(x)\}$ is a closed subset in $A \times Y$. 
Definition 2 [27] Let $X, Y$ be two topological vector spaces, $A$ be a nonempty subset of $X, F: A \rightarrow 2^{Y}$ be a multifunction and $C \subset Y$ be a nonempty closed convex cone.

(i) $F$ is called upper $C$-continuous at $x_{0} \in A$ if for any neighborhood $U$ of the origin in $Y$, there is a neighborhood $V$ of $x_{0}$ such that

$$
F(x) \subset F\left(x_{0}\right)+U+C, \quad \forall x \in V .
$$

(ii) $F$ is called lower $C$-continuous at $x_{0} \in A$ if for any neighborhood $U$ of the origin in $Y$, there is a neighborhood $V$ of $x_{0}$ such that

$$
F\left(x_{0}\right) \subset F(x)+U-C, \quad \forall x \in V .
$$

Definition 3 [6] Let $X, Y$ be two topological vector spaces and $A$ be a nonempty subset of $X$ and $C \subset Y$ be a nonempty closed convex cone. A set-valued mapping $F: A \rightarrow 2^{Y}$ is said to be type II $C$-lower semicontinuous at $x_{0} \in A$ if for each $y \in F\left(x_{0}\right)$ and any neighborhood $U$ of the origin in $Y$, there exists a neighborhood $U\left(x_{0}\right)$ of $x_{0}$ such that

$$
F(x) \cap(y+U-C) \neq \emptyset, \quad \forall x \in U\left(x_{0}\right) \cap A .
$$

Definition $4[6,28]$ Let $X$ and $Y$ be two topological vector spaces and $A$ be a nonempty convex subset of $X$. A set-valued mapping $F: A \rightarrow 2^{Y}$ is said to be $C$-convex if for any $x, y \in A$ and $t \in[0,1]$, one has

$$
F(t x+(1-t) y) \subset t F(x)+(1-t) F(y)-C .
$$

$F$ is said to be $C$-concave if $-F$ is $C$-convex.

Definition 5 [28] Let $X$ and $Y$ be two topological vector spaces and $A$ be a nonempty convex subset of $X$. A set-valued mapping $F: A \rightarrow 2^{Y}$ is said to be properly $C$-quasiconvex if for any $x, y \in A$ and $t \in[0,1]$, we have

$$
\begin{gathered}
\text { either } \quad F(x) \subset F(t x+(1-t) y)+C, \\
\text { or } \quad F(y) \subset F(t x+(1-t) y)+C .
\end{gathered}
$$

Lemma 6 [28] Let $X, Y$ be two topological vector spaces, $A$ be a nonempty convex subset of $X$ and $F: A \rightarrow 2^{Y}$ be a multifunction.

(i) If $F$ is upper semicontinuous at $x_{0} \in A$ with closed values, then $F$ is closed at $x_{0} \in A$;

(ii) If $F$ is closed at $x_{0} \in A$ and $Y$ is compact, then $F$ is upper semicontinuous at $x_{0} \in A$.

(iii) If $F$ has compact values, then $F$ is usc at $x_{0} \in A$ if and only if, for each net $\left\{x_{\alpha}\right\} \subseteq A$ which converges to $x_{0} \in A$ and for each net $\left\{y_{\alpha}\right\} \subseteq F\left(x_{\alpha}\right)$, there are $y_{0} \in F\left(x_{0}\right)$ and a subnet $\left\{y_{\beta}\right\}$ of $\left\{y_{\alpha}\right\}$ such that $y_{\beta} \rightarrow y_{0}$.

Lemma 7 (Kakutani-Fan-Glickcberg (see [29])) Let A be a nonempty compact convex subset of a locally convex Hausdorff vector topological space $X$. If $F: A \longrightarrow 2^{A}$ is upper semicontinuous and for any $x \in A, F(x)$ is nonempty, convex and closed, then there exists an $x^{\prime \prime} \in A$ such that $x^{*} \in F\left(x^{*}\right)$. 


\section{Main results}

In this section, we discuss the existence and closedness of the solution sets of symmetric generalized quasi-variational inclusion problems by using the Kakutani-Fan-Glicksberg fixed point theorem.

Theorem 8 For each $\{i=1,2\}$, assume for the problem $\left(\mathrm{SQVIP}_{\alpha}\right)$ that

(i) $K_{i}$ is usc in $A \times A$ with nonempty convex closed values and $P_{i}$ is lsc in $A \times A$ with nonempty closed values;

(ii) $T_{i}$ is usc in $A \times A$ with nonempty convex compact values if $\alpha=w($ or $\alpha=m)$ and $T_{i}$ is lsc in $A \times A$ with nonempty convex values if $\alpha=s$;

(iii) for all $(x, z, u) \in A \times B \times A, 0 \in F_{i}\left(x, z, P_{i}(x, u)\right)$;

(iv) for all $(x, z, u) \in A \times B \times A$, the set $\left\{a \in K_{i}(x, u): 0 \in F_{i}(a, z, y), \forall y \in P_{i}(x, u)\right\}$ is convex:

(v) the set $\left\{(x, z, y) \in A \times B \times A: 0 \in F_{i}(x, z, y)\right\}$ is closed.

Then the $\left(\mathrm{SQVIP}_{\alpha}\right)$ has a solution, i.e., there exist $(\bar{x}, \bar{u}) \in A \times A$ such that $\bar{x} \in K_{1}(\bar{x}, \bar{u})$, $\bar{u} \in K_{2}(\bar{x}, \bar{u})$ and

$$
\begin{aligned}
& (y, \bar{z}) \alpha P_{1}(\bar{x}, \bar{u}) \times T_{1}(\bar{x}, \bar{u}) \quad \text { satisfying } 0 \in F_{1}(\bar{x}, \bar{z}, y), \\
& (y, \bar{v}) \alpha P_{2}(\bar{x}, \bar{u}) \times T_{2}(\bar{x}, \bar{u}) \quad \text { satisfying } 0 \in F_{2}(\bar{u}, \bar{v}, y) .
\end{aligned}
$$

Moreover, the solution set of the $\left(\mathrm{SQVIP}_{\alpha}\right)$ is closed.

Proof Similar arguments can be applied to three cases. We present only the proof for the case where $\alpha=m$.

Indeed, for all $(x, z, u, v) \in A \times B \times A \times B$, define mappings: $\Phi_{m}, \Pi_{m}: A \times B \times A \rightarrow 2^{A}$ by

$$
\Phi_{m}(x, z, u)=\left\{a \in K_{1}(x, u): 0 \in F_{1}(a, z, y), \forall y \in P_{1}(x, u)\right\},
$$

and

$$
\Pi_{m}(x, v, u)=\left\{b \in K_{2}(x, u): 0 \in F_{2}(b, v, y), \forall y \in P_{2}(x, u)\right\} .
$$

(a) Show that $\Phi_{m}(x, z, u)$ and $\Pi_{m}(x, v, u)$ are nonempty convex sets. Indeed, for all $(x, z, u) \in A \times B \times A$ and $(x, v, u) \in A \times B \times A$, for each $\{i=1,2\}$, $K_{i}(x, u), P_{i}(x, u)$ are nonempty. Thus, by assumptions (i), (ii) and (iii), we have $\Phi_{m}(x, z, u)$ and $\Pi_{m}(x, v, u)$ are nonempty. On the other hand, by the condition (iv), we also have $\Phi_{m}(x, z, u), \Pi_{m}(x, v, u)$ are convex.

(b) We will prove $\Phi_{m}$ and $\Pi_{m}$ are upper semicontinuous in $A \times B \times A$ with nonempty closed values.

First, we show that $\Phi_{m}$ is upper semicontinuous in $A \times B \times A$ with nonempty closed values. Since $A$ is a compact set, by Lemma 6(ii), we need only to show that $\Phi_{m}$ is a closed mapping. Indeed, let a net $\left\{\left(x_{n}, z_{n}, u_{n}\right): n \in I\right\} \subset A \times B \times A$ such that $\left(x_{n}, z_{n}, u_{n}\right) \rightarrow(x, z, u) \in A \times B \times A$, and let $a_{n} \in \Phi_{m}\left(x_{n}, z_{n}, u_{n}\right)$ such that $a_{n} \rightarrow a_{0}$. Now we need to show that $a_{0} \in \Phi_{m}(x, z, u)$. Since $a_{n} \in K_{1}\left(x_{n}, u_{n}\right)$ and $K_{1}$ is upper semicontinuous with nonempty closed values by Lemma $6(\mathrm{i})$, hence $K_{1}$ is closed, 
thus we have $a_{0} \in K_{1}(x, u)$. Suppose the contrary $a_{0} \notin \Phi_{m}(x, z, u)$. Then $\exists y_{0} \in P_{1}(x, u)$ such that

$$
0 \notin F_{1}\left(a_{0}, z, y_{0}\right) .
$$

By the lower semicontinuity of $P_{1}$, there is a net $\left\{y_{n}\right\}$ such that $y_{n} \in P_{1}\left(x_{n}, u_{n}\right)$, $y_{n} \rightarrow y_{0}$. Since $a_{n} \in \Phi_{m}\left(x_{n}, z_{n}, u_{n}\right)$, we have

$$
0 \in F_{1}\left(a_{n}, z_{n}, y_{n}\right)
$$

By the condition (v) and (2), we have

$$
0 \in F_{1}\left(a_{0}, z, y_{0}\right) \text {. }
$$

This is a contradiction between (3) and (1). Thus, $a_{0} \in \Phi_{m}(x, z, u)$. Hence, $\Phi_{m}$ is upper semicontinuous in $A \times B \times A$ with nonempty closed values. Similarly, we also have $\Pi_{m}(x, v, u)$ is upper semicontinuous in $A \times B \times A$ with nonempty closed values.

(c) Now we need to prove the solution set $\Xi_{m}(F) \neq \emptyset$.

Define the set-valued mappings $\Theta_{m}, \Omega_{m}: A \times B \times A: \rightarrow 2^{A \times B}$ by

$$
\Theta_{m}(x, z, u)=\left(\Phi_{m}(x, z, u), T_{1}(x, u)\right), \quad \forall(x, z, u) \in A \times B \times A
$$

and

$$
\Omega_{m}(x, v, u)=\left(\Pi_{m}(x, v, u), T_{2}(x, u)\right), \quad \forall(x, v, u) \in A \times B \times A .
$$

Then $\Theta_{m}, \Omega_{m}$ are upper semicontinuous and $\forall(x, z, u) \in A \times B \times A$, $\forall(x, v, u) \in A \times B \times A, \Theta_{m}(x, z, u)$ and $\Theta_{m}(x, v, u)$ are nonempty closed convex subsets of $A \times B \times A$.

Define the set-valued mapping $H:(A \times B) \times(A \times B) \rightarrow 2^{(A \times B) \times(A \times B)}$ by

$$
H((x, z),(u, v))=\left(\Theta_{m}(x, z, u), \Omega_{m}(x, v, u)\right), \quad \forall((x, z),(u, v)) \in(A \times B) \times(A \times B) .
$$

Then $H$ is also upper semicontinuous and $\forall((x, z),(u, v)) \in(A \times B) \times(A \times B)$, $H((x, z),(u, v))$ is a nonempty closed convex subset of $(A \times B) \times(A \times B)$.

By Lemma 7, there exists a point $\left(\left(x^{*}, z^{*}\right),\left(v^{*}, u^{*}\right)\right) \in(A \times B) \times(A \times B)$ such that $\left(\left(x^{*}, z^{\prime \prime}\right),\left(u^{*}, v^{*}\right)\right) \in H\left(\left(x^{\prime \prime}, z^{*}\right),\left(u^{\prime \prime}, v^{*}\right)\right)$, that is,

$$
\left(x^{*}, z^{*}\right) \in \Theta_{m}\left(x^{*}, z^{*}, u^{*}\right), \quad\left(u^{*}, v^{*}\right) \in \Omega_{m}\left(x^{*}, v^{*}, u^{*}\right),
$$

which implies that $x^{*} \in \Phi_{m}\left(x^{*}, z^{*}, u^{*}\right), z^{*} \in T_{1}\left(x^{*}, u^{*}\right), u^{*} \in \Pi_{m}\left(x^{*}, v^{*}, u^{*}\right)$ and $v^{*} \in T_{2}\left(x^{*}, u^{*}\right)$. Hence, there exists $\left(x^{*}, u^{*}\right) \in A \times A, z^{*} \in T_{1}\left(x^{*}, u^{*}\right), v^{*} \in T_{2}\left(x^{*}, u^{*}\right)$ such that $x^{*} \in K_{1}\left(x^{*}, u^{\prime \prime}\right), u^{*} \in K_{2}\left(x^{*}, u^{*}\right)$, satisfying

$$
0 \in F_{1}\left(x^{*}, z^{*}, y\right), \quad \forall y \in P_{1}\left(x^{*}, u^{*}\right)
$$


and

$$
0 \in F_{2}\left(u^{*}, v^{*}, y\right), \quad \forall y \in P_{2}\left(x^{*}, u^{*}\right)
$$

i.e., $\left(\mathrm{SQVIP}_{\alpha}\right)$ has a solution.

(d) Now we prove that $\Xi_{m}(F)$ is closed. Indeed, let a net $\left\{\left(x_{n}, u_{n}\right), n \in I\right\} \in \Xi_{m}(F)$ : $\left(x_{n}, u_{n}\right) \rightarrow\left(x_{0}, u_{0}\right)$. We need to prove that $\left(x_{0}, u_{0}\right) \in \Xi_{m}(F)$. Indeed, by the lower semicontinuity of $P_{i}, i=1,2$, for any $y_{0} \in P_{i}\left(x_{0}, u_{0}\right)$, there exists $y_{n} \in P_{i}\left(x_{n}, u_{n}\right)$ such that $y_{n} \rightarrow y_{0}$. Since $\left(x_{n}, u_{n}\right) \in \Xi_{m}(F)$, there exists $z_{n} \in T_{1}\left(x_{n}, u_{n}\right), v_{n} \in T_{2}\left(x_{n}, u_{n}\right)$, $x_{n} \in K_{1}\left(x_{n}, u_{n}\right), u_{n} \in K_{2}\left(x_{n}, u_{n}\right)$ such that

$$
0 \in F_{1}\left(x_{n}, z_{n}, y_{n}\right)
$$

and

$$
0 \in F_{2}\left(u_{n}, v_{n}, y_{n}\right)
$$

Since $K_{1}, K_{2}$ are upper semicontinuous in $A \times A$ with nonempty closed values, by Lemma 6(i), we have $K_{1}, K_{2}$ are closed. Thus, $x_{0} \in K_{1}\left(x_{0}, u_{0}\right), u_{0} \in K_{2}\left(x_{0}, u_{0}\right)$. Since $T_{1}, T_{2}$ are upper semicontinuous in $A \times A$ with nonempty compact values, there exists $z_{0} \in T_{1}\left(x_{0}, u_{0}\right)$ and $v_{0} \in T_{2}\left(x_{0}, u_{0}\right)$ such that $z_{n} \rightarrow z_{0}, v_{n} \rightarrow v_{0}$ (taking subnets if necessary). By the condition (v) and $\left(x_{n}, z_{n}, u_{n}, v_{n}\right) \rightarrow\left(x_{0}, z_{0}, u_{0}, v_{0}\right)$, we have

$$
0 \in F_{1}\left(x_{0}, z_{0}, y_{0}\right)
$$

and

$$
0 \in F_{2}\left(u_{0}, v_{0}, y_{0}\right) \text {. }
$$

This means that $\left(x_{0}, u_{0}\right) \in \Xi_{m}(F)$. Thus, $\Xi_{m}(F)$ is a closed set.

If $K_{1}(x, u)=P_{1}(x, u)=S_{1}(x, u), K_{2}(x, u)=P_{2}(x, u)=S_{2}(x, u), \alpha=m$, and $F_{1}(x, z, y)=$ $G_{1}(x, z, y)-C, F_{2}(u, z, y)=G_{2}(u, z, y)-C$, with $S_{1}, S_{2}: A \times A \rightarrow 2^{A}, G_{1}, G_{2}: A \times B \times A \rightarrow 2^{Z}$ are set-valued mappings, and $C \subset Z$ is a nonempty closed convex cone. Then (SQVIP $\left.{ }_{\alpha}\right)$ becomes (SSVQEP) studied in [6].

In this special case, we have the following corollary.

\section{Corollary 9 For each $\{i=1,2\}$, assume for the problem (SSVQEP) that}

(i) $S_{i}$ is continuous in $A \times A$ with nonempty convex closed values;

(ii) $T_{i}$ is usc in $A \times A$ with nonempty convex compact values;

(iii) for all $(x, z, u) \in A \times B \times A, G_{i}\left(x, z, S_{i}(x, u)\right) \subset C$;

(iv) for all $(x, z, u) \in A \times B \times A$, the set $\left\{a \in S_{i}(x, u): G_{i}(a, z, y) \subset C, \forall y \in S_{i}(x, u)\right\}$ is convex;

(v) the set $\left\{(x, z, y) \in A \times B \times A: G_{i}(x, z, y) \subset C\right\}$ is closed.

Then the (SSVQEP) has a solution, i.e., there exist $(\bar{x}, \bar{u}) \in A \times A$ and $\bar{z} \in T_{1}(\bar{x}, \bar{u}), \bar{v} \in$ $T_{2}(\bar{x}, \bar{u})$ such that $\bar{x} \in S_{1}(\bar{x}, \bar{u}), \bar{u} \in S_{2}(\bar{x}, \bar{u})$ and

$$
G_{1}(\bar{x}, \bar{z}, y) \subset C, \quad \forall y \in S_{1}(\bar{x}, \bar{u})
$$


and

$$
G_{2}(\bar{u}, \bar{v}, y) \subset C, \quad \forall y \in S_{2}(\bar{x}, \bar{u}) .
$$

Moreover, the solution set of the (SSVQEP) is closed.

Remark 10 Chen et al. [6] obtained an existence result of (SSVQEP). However, the assumptions in Theorem 3.1 in [6] are different from the assumptions in Corollary 9. The following example shows that all assumptions of Corollary 9 are satisfied. But Theorem 3.1 in [6] is not fulfilled.

Example 11 Let $X=Y=Z=\mathbb{R}, A=B=[0,1], C=[0,+\infty)$ and let $S_{1}(x)=S_{2}(x)=[0,1]$, $G_{1}, G_{2}, F:[0,1] \times[0,1] \times[0,1] \rightarrow 2^{\mathbb{R}}$ and

$$
T_{1}(x, u)=T_{2}(x, u)= \begin{cases}{[0,2]} & \text { if } x_{0}=u_{0}=\frac{1}{2} \\ {[0,1]} & \text { otherwise }\end{cases}
$$

and

$$
G_{1}(x, z, y)=G_{2}(u, z, y)=F(x, z, y)= \begin{cases}{\left[\frac{1}{2}, 1\right]} & \text { if } x_{0}=z_{0}=y_{0}=\frac{1}{2}, \\ {[1,2]} & \text { otherwise. }\end{cases}
$$

We show that assumptions of Corollary 9 are easily seen to be fulfilled. Hence, by Corollary 9, (SSVQEP) has a solution. But $F$ is neither type II $C$-lower semicontinuous nor $C$-concave at $x_{0}=\frac{1}{2}$. Thus, Theorem 3.1 in [6] does not work.

If $K_{1}(x, u)=P_{1}(x, u)=S_{1}(x), K_{2}(x, u)=P_{2}(x, u)=S_{2}(u), T_{1}(x, u)=T_{1}(x), T_{2}(x, u)=T_{2}(u)$, $\alpha=m$ and $F_{1}(x, z, y)=G_{1}(x, z, y)-C, F_{2}(u, z, y)=G_{2}(u, z, y)-C$, with $S_{1}, S_{2}: A \rightarrow 2^{A}, G_{1}, G_{2}$ : $A \times B \times A \rightarrow 2^{Z}$ are set-valued mappings, and $C \subset Z$ is a nonempty closed convex cone. Then $\left(\mathrm{SQVIP}_{\alpha}\right)$ becomes (SGSVQEP) studied in [5].

In this special case, we have the following corollary.

Corollary 12 For each $\{i=1,2\}$, assume for the problem (SGSVQEP) that

(i) $S_{i}$ is continuous in A with nonempty convex closed values;

(ii) $T_{i}$ is usc in $A$ with nonempty convex compact values;

(iii) for all $(x, z) \in A \times B, G_{i}\left(x, z, S_{i}(x)\right) \subset C$;

(iv) for all $(x, z) \in A \times B$, the set $\left\{a \in S_{i}(x): G_{i}(a, z, y) \subset C, \forall y \in S_{i}(x)\right\}$ is convex;

(v) the set $\left\{(x, z, y) \in A \times B \times A: G_{i}(x, z, y) \subset C\right\}$ is closed.

Then the (SGSVQEP) has a solution, i.e., there exist $(\bar{x}, \bar{u}) \in A \times A$ and $\bar{z} \in T_{1}(\bar{x}), \bar{v} \in T_{2}(\bar{u})$ such that $\bar{x} \in S_{1}(\bar{x}), \bar{u} \in S_{2}(\bar{u})$ and

$$
G_{1}(\bar{x}, \bar{z}, y) \subset C, \quad \forall y \in S_{1}(\bar{x})
$$

and

$$
G_{2}(\bar{u}, \bar{v}, y) \subset C, \quad \forall y \in S_{2}(\bar{u})
$$


Remark 13 In [5], Plubtieng-Sitthithakerngkiet also obtained an existence result of (SGSVQEP). However, the assumptions in Theorem 3.1 in [5] are different from the assumptions in Corollary 12. The following example shows that in this special case, all assumptions of Corollary 12 are satisfied. But Theorem 3.1 in [5] is not fulfilled.

Example 14 Let $X=Y=Z=\mathbb{R}, A=B=[0,1], C=[0,+\infty)$ and let $S_{1}(x)=S_{2}(x)=[0,1]$, $F:[0,1] \times[0,1] \times[0,1] \rightarrow 2^{\mathbb{R}}$ and

$$
T_{1}(x)=T_{2}(x)= \begin{cases}{[0,2]} & \text { if } x_{0}=\frac{1}{2} \\ {[0,1]} & \text { otherwise }\end{cases}
$$

and

$$
G_{1}(x, z, y)=G_{2}(u, z, y)=F(x, z, y)= \begin{cases}{\left[\frac{1}{2}, 1\right]} & \text { if } x_{0}=z_{0}=y_{0}=\frac{1}{2} \\ {[1,2]} & \text { otherwise. }\end{cases}
$$

We show that all assumptions of Corollary 12 are satisfied. So, by this corollary, the considered problem has solutions. However, $F$ is not lower $(-C)$-continuous at $x_{0}=\frac{1}{2}$. Also, Theorem 3.1 in [5] does not work.

If $K_{1}(\bar{x}, \bar{u})=P_{1}(\bar{x}, \bar{u})=K_{2}(\bar{x}, \bar{u})=P_{2}(\bar{x}, \bar{u})=S(\bar{x}), T_{1}(\bar{x}, \bar{u})=T_{2}(\bar{x}, \bar{u})=\{z\}$ and $F_{1}(x, z, y)=$ $F_{2}(u, z, y)=G(x, y)-C$, for each $\bar{x}, \bar{u} \in A$ and $S: A \rightarrow 2^{A}, G: A \times A \rightarrow 2^{Z}$ are setvalued mappings, and $C \subset Z$ is a nonempty closed convex cone. Then $\left(\operatorname{SQVIP}_{\alpha}\right)$ becomes (SVQEP) studied in [21].

In this special case, we also have the following corollary.

Corollary 15 Assume for the problem (SVQEP) that

(i) $S$ is continuous in A with nonempty convex closed values;

(ii) for all $x \in A, G(x, S(x)) \subset C$;

(iii) for all $x \in A$, the set $\{a \in S(x): G(a, y) \subset C, \forall y \in S(x)\}$ is convex;

(iv) the set $\{(x, y) \in A \times A: G(x, y) \subset C\}$ is closed.

Then the (SVQEP) has a solution, i.e., there exists $\bar{x} \in S(\bar{x})$ such that

$$
G(\bar{x}, y) \subset C, \quad \forall y \in S(\bar{x}) .
$$

Moreover, the solution set of the (SVQEP) is closed.

The following example shows that in this special case, all assumptions of Corollary 15 are satisfied. But Theorem 3.3 in [21] is not fulfilled.

Example 16 Let $X, Y, Z, A, B, C$ as in Example 14, and let $S(x)=[0,1], G:[0,1] \times[0,1] \rightarrow$ $2^{\mathbb{R}}$ and

$$
G(x, y)= \begin{cases}{\left[1, \frac{5}{2}\right]} & \text { if } x_{0}=z_{0}=y_{0}=\frac{1}{2} \\ {\left[\frac{1}{5}, \frac{3}{4}\right]} & \text { otherwise. }\end{cases}
$$

We show that all assumptions of Corollary 15 are satisfied. So, (SVQEP) has a solution. However, $G$ is not upper $C$-continuous at $x_{0}=\frac{1}{2}$. Also, Theorem 3.3 in [21] does not work. 
The following example shows that all assumptions of Corollary 9, Corollary 12 and Corollary 15 are satisfied. However, Theorem 3.1 in [6], Theorem 3.1 in [5] and Theorem 3.3 in [21] are not fulfilled. The reason is that $G$ is not properly $C$-quasiconvex.

Example 17 Let $A, B, X, Y, Z, C$ as in Example 14, and let $S:[0,1] \rightarrow 2^{\mathbb{R}}, G:[0,1] \times$ $[0,1] \rightarrow 2^{\mathbb{R}}, S_{1}(x, u)=S_{2}(x, u)=S(x)=[0,1], T_{1}(x, u)=T_{2}(x, u)=T(x, u)=\{z\}$ and

$$
G_{1}(x, z, y)=G_{2}(u, z, y)=G(x, y)= \begin{cases}{[1,2]} & \text { if } x_{0}=y_{0}=\frac{1}{2} \\ {\left[\frac{1}{2}, 1\right]} & \text { otherwise }\end{cases}
$$

We show that all assumptions of Corollary 9, Corollary 12 and Corollary 15 are satisfied. However, $G$ is not properly $C$-quasiconvex at $x_{0}=\frac{1}{2}$. Thus, it gives the case where Corollary 9, Corollary 12 and Corollary 15 can be applied but Theorem 3.1 in [6], Theorem 3.1 in [5] and Theorem 3.3 in [21] do not work.

\section{Applications}

Since our symmetric vector quasi-equilibrium problems include many rather general problems as particular cases mentioned in Section 1, from the results of Section 2 we can derive consequences for such special cases. In this section, we discuss only some corollaries for symmetric weak and strong quasi-equilibrium problems as examples.

Let $X, Y, Z, A, B$ be as in Section 1, and $C \subset Z$ be a nonempty closed convex cone. Let $S_{i}, P_{i}: A \times A \rightarrow 2^{A}, T_{i}: A \times A \rightarrow 2^{B}$ be set-valued mappings and $f_{i}: A \times B \times A \rightarrow Z$, $i=1,2$ be vector-valued functions. We consider the two following symmetric weak and strong vector quasi-equilibrium problems (in short, (SWQVEP) and (SSQVEP)), respectively.

(SWQVEP): Find $(\bar{x}, \bar{u}) \in A \times A$ and $\bar{z} \in T_{1}(\bar{x}, \bar{u}), \bar{v} \in T_{2}(\bar{x}, \bar{u})$ such that $\bar{x} \in S_{1}(\bar{x}, \bar{u}), \bar{u} \in$ $S_{2}(\bar{x}, \bar{u})$ satisfying

$$
\begin{aligned}
& f_{1}(\bar{x}, \bar{z}, y) \notin-\operatorname{int} C, \quad \forall y \in S_{1}(\bar{x}, \bar{u}), \\
& f_{2}(\bar{u}, \bar{v}, y) \notin-\operatorname{int} C, \quad \forall y \in S_{2}(\bar{x}, \bar{u}) .
\end{aligned}
$$

(SSQVEP): Find $(\bar{x}, \bar{u}) \in A \times A$ and $\bar{z} \in T_{1}(\bar{x}, \bar{u}), \bar{v} \in T_{2}(\bar{x}, \bar{u})$ such that $\bar{x} \in S_{1}(\bar{x}, \bar{u}), \bar{u} \in$ $S_{2}(\bar{x}, \bar{u})$ satisfying

$$
\begin{aligned}
& f_{1}(\bar{x}, \bar{z}, y) \in C, \quad \forall y \in S_{1}(\bar{x}, \bar{u}), \\
& f_{2}(\bar{u}, \bar{v}, y) \in C, \quad \forall y \in S_{2}(\bar{x}, \bar{u}) .
\end{aligned}
$$

Corollary 18 For each $\{i=1,2\}$, assume for the problem (SWQVEP) that

(i) $S_{i}$ is continuous in $A \times A$ with nonempty convex closed values;

(ii) $T_{i}$ is usc in $A \times A$ with nonempty convex compact values;

(iii) for all $(x, z, u) \in A \times B \times A, f_{i}\left(x, z, S_{i}(x, u)\right) \notin-$ int $C$;

(iv) for all $(x, z, u) \in A \times B \times A$, the set $\left\{a \in S_{i}(x, u): f_{i}(a, z, y) \notin-\operatorname{int} C, \forall y \in S_{i}(x, u)\right\}$ is convex;

(v) the $\operatorname{set}\left\{(x, z, y) \in A \times B \times A: f_{i}(x, z, y) \notin-\right.$ int $\left.C\right\}$ is closed. 
Then the (SWQVEP) has a solution, i.e., there exist $(\bar{x}, \bar{u}) \in A \times A$ and $\bar{z} \in T_{1}(\bar{x}, \bar{u}), \bar{v} \in$ $T_{2}(\bar{x}, \bar{u})$ such that $\bar{x} \in S_{1}(\bar{x}, \bar{u}), \bar{u} \in S_{2}(\bar{x}, \bar{u})$ satisfying

$$
\begin{aligned}
& f_{1}(\bar{x}, \bar{z}, y) \notin-\operatorname{int} C, \quad \forall y \in S_{1}(\bar{x}, \bar{u}), \\
& f_{2}(\bar{u}, \bar{v}, y) \notin-\operatorname{int} C, \quad \forall y \in S_{2}(\bar{x}, \bar{u}) .
\end{aligned}
$$

Moreover, the solution set of the (SWQVEP) is closed.

Proof Setting $\alpha=m, F_{1}(x, z, y)=Z \backslash\left(f_{1}(x, z, y)+\operatorname{int} C\right)$ and $F_{2}(u, z, y)=Z \backslash\left(f_{2}(u, z, y)+\operatorname{int} C\right)$, problem (SWQVEP) becomes a particular case of $\left(\mathrm{SQVIP}_{\alpha}\right)$ and Corollary 18 is a direct consequence of Theorem 8 .

Corollary 19 Assume for the problem (SWQVEP) assumptions (i), (ii), (iii) and (iv) as in Corollary 18 and replace $(\mathrm{v})$ by $\left(\mathrm{v}^{\prime}\right)$

$\left(\mathrm{v}^{\prime}\right)$ for each $i=\{1,2\}, f_{i}$ is continuous in $A \times B \times A$.

Then the (SWQVEP) has a solution. Moreover, the solution set of the (SWQVEP) is closed.

Proof We omit the proof since the technique is similar to that for Corollary 18 with suitable modifications.

Corollary 20 For each $\{i=1,2\}$, assume for the problem (SSQVEP) that

(i) $S_{i}$ is continuous in $A \times A$ with nonempty convex closed values;

(ii) $T_{i}$ is usc in $A \times A$ with nonempty convex compact values;

(iii) for all $(x, z, u) \in A \times B \times A, f_{i}\left(x, z, S_{i}(x, u)\right) \in C$;

(iv) for all $(x, z, u) \in A \times B \times A$, the set $\left\{a \in S_{i}(x, u): f_{i}(a, z, y) \in C, \forall y \in S_{i}(x, u)\right\}$ is convex;

(v) the set $\left\{(x, z, y) \in A \times B \times A: f_{i}(x, z, y) \in C\right\}$ is closed.

Then the (SSQVEP) has a solution, i.e., there exist $(\bar{x}, \bar{u}) \in A \times A$ and $\bar{z} \in T_{1}(\bar{x}, \bar{u}), \bar{v} \in$ $T_{2}(\bar{x}, \bar{u})$ such that $\bar{x} \in S_{1}(\bar{x}, \bar{u}), \bar{u} \in S_{2}(\bar{x}, \bar{u})$ satisfying

$$
\begin{aligned}
& f_{1}(\bar{x}, \bar{z}, y) \in C, \quad \forall y \in S_{1}(\bar{x}, \bar{u}), \\
& f_{2}(\bar{u}, \bar{v}, y) \in C, \quad \forall y \in S_{2}(\bar{x}, \bar{u}) .
\end{aligned}
$$

Moreover, the solution set of the (SSQVEP) is closed.

Proof Setting $\alpha=m, F_{1}(x, z, y)=f_{1}(x, z, y)-C$ and $F_{2}(u, z, y)=f_{2}(u, z, y)-C$, problem (SSQVEP) becomes a particular case of $\left(\operatorname{SQVIP}_{\alpha}\right)$ and the Corollary 20 is a direct consequence of Theorem 8 .

Corollary 21 Assume for the problem (SSQVEP) assumptions (i), (ii), (iii) and (iv) as in Corollary 20 and replace $(\mathrm{v})$ by $\left(\mathrm{v}^{\prime}\right)$

$\left(\mathrm{v}^{\prime}\right)$ for each $i=\{1,2\}, f_{i}$ is continuous in $A \times B \times A$.

Then the (SSQVEP) has a solution. Moreover, the solution set of the (SSQVEP) is closed. 


\section{Acknowledgements}

The author thanks the two anonymous referees for their valuable remarks and suggestions, which helped them to improve the article considerably.

Received: 5 July 2012 Accepted: 9 January 2013 Published: 6 February 2013

\section{References}

1. Ansari, QH, Oettli, W, Schiager, D: A generalization of vectorial equilibria. Math. Methods Oper. Res. 46, 147-152 (1997)

2. Ansari, QH, Schaible, S, Yao, JC: System of generalized vector equilibrium problems with applications. J. Glob. Optim. 22, 3-16 (2002)

3. Ansari, QH, Konnov, IV, Yao, JC: On generalized vector equilibrium problems. Nonlinear Anal. TMA 47, 543-554 (2001)

4. Long, XJ, Huang, NJ, Teo, KL: Existence and stability of solutions for generalized strong vector quasi-equilibrium problems. Math. Comput. Model. 47, 445-451 (2008)

5. Plubtieng, S, Sitthithakerngkiet, $\mathrm{K}$ : On the existence result for system of generalized strong vector quasi-equilibrium problems. Fixed Point Theory Appl. 2011, Article ID 475121 (2011)

6. Chen, B, Huang, NJ, Cho, YJ: Symmetric strong vector quasiequilibrium problems in Hausdorff locally convex spaces. J. Inequal. Appl. 2011, 56 (2011)

7. Hung, NV: Sensitivity analysis for generalized quasi-variational relation problems in locally G-convex spaces. Fixed Point Theory Appl. 2012, 158 (2012)

8. Hung, NV: Continuity of solutions for parametric generalized quasi-variational relation problems. Fixed Point Theory Appl. 2012, 102 (2012)

9. Anh, LQ, Khanh, PQ: On the stability of the solution sets of general multivalued vector quasiequilibrium problems. J. Optim. Theory Appl. 135, 271-284 (2007)

10. Anh, LQ, Khanh, $\mathrm{PQ}$ : Existence conditions in symmetric multivalued vector quasiequilibrium problem. Contro Cybern. 36, 519-530 (2008)

11. Fu, JY: Symmetric vector quasiequilibrium problems. J. Math. Anal. Appl. 285, 708-713 (2003)

12. Lin, L, Liu, YH: Existence theorems for systems of generalized vector quasiequilibrium problems and optimization problems. J. Optim. Theory Appl. 130, 461-475 (2006)

13. Kien, BT, Huy, NQ, Wong, NC: On the solution existence of generalized vector quasi-equilibrium problems with discontinuous multifunctions. Taiwan. J. Math. 13, 757-775 (2009)

14. Liu, QY, Long, XJ, Huang, NJ: Connectedness of the sets of weak efficient solutions for generalized vector equilibrium problems. Math. Slovaca 62, 123-136 (2012)

15. Long, XJ, Peng, JW: Connectedness and compactness of weak efficient solutions for vector equilibrium problems. Bull. Korean Math. Soc. 48, 1225-1233 (2011)

16. Long, XJ, Huang, YQ, Peng, ZY: Optimality conditions for the Henig efficient solution of vector equilibrium problems with constraints. Optim. Lett. 5, 717-728 (2011)

17. Lin, Z, Yang, $H, Y u, J$ : On existence and essential components of the solution set for the system of vector quasiequilibrium problems. Nonlinear Anal. 63, 2445-2452 (2005)

18. Lin, Z, Yu, J: The existence of solutions for the system of generalized vector quasiequilibrium problems. Appl. Math. Lett. 18, 415-422 (2005)

19. Song, QQ, Wang, LS: The existence of solutions for the system of vector quasiequilibrium problems in topological order spaces. Comput. Math. Appl. 62, 1979-1983 (2011)

20. Plubtieng, S, Sitthithakerngkiet, K: Existence result of generalized vector quasiequilibrium problems in locally G-convex spaces. Fixed Point Theory Appl. 2011, Article ID 967515 (2011)

21. Yang, Y, Pu, YJ: On the existence and essential components for solution set for system of strong vector quasiequilibrium problems. J. Glob. Optim. (2011). doi:10.1007/s10898-011-9830-y

22. Blum, E, Oettli, W: From optimization and variational inequalities to equilibrium problems. Math. Stud. 63, 123-145 (1994)

23. Hou, SH, Gong, XH, Yang, XM: Existence and stability of solutions for generalized Ky Fan inequality problems with trifunctions. J. Optim. Theory Appl. 146, 387-398 (2010)

24. Lin, Z: Existence of solutions to the system of generalized implicit vector quasivariational inequality problems. Fixed Point Theory Appl. 2009, Article ID 654370 (2009)

25. Balaj, M, Lin, LJ: Generalized variational relation problems with applications. J. Optim. Theory Appl. 148, 1-13 (2011)

26. Yu, J: Essential weak efficient solution in multiobjective optimization problems. J. Math. Anal. Appl. 166, 230-235 (1992)

27. Berge, C: Topological Spaces. Oliver and Boyd, London (1963)

28. LUC, DT: Theory of Vector Optimization: Lecture Notes in Economics and Mathematical Systems. Springer, Berlin (1989)

29. Holmes, RB: Geometric Functional Analysis and Its Application. Springer, New York (1975)

doi:10.1186/1029-242X-2013-40

Cite this article as: Hung: Existence conditions for symmetric generalized quasi-variational inclusion problems.

Journal of Inequalities and Applications 2013 2013:40. 